\title{
Review Article \\ Fosfomycin: A First-Line Oral Therapy for Acute Uncomplicated Cystitis
}

\author{
George G. Zhanel, Andrew J. Walkty, and James A. Karlowsky \\ Department of Medical Microbiology, College of Medicine, University of Manitoba, Winnipeg, MB, Canada R3A 1R9 \\ Correspondence should be addressed to George G. Zhanel; ggzhanel@pcs.mb.ca
}

Received 13 November 2015; Accepted 31 March 2016

Academic Editor: Daniele Focosi

Copyright ( 2016 George G. Zhanel et al. This is an open access article distributed under the Creative Commons Attribution License, which permits unrestricted use, distribution, and reproduction in any medium, provided the original work is properly cited.

\begin{abstract}
Fosfomycin is a new agent to Canada approved for the treatment of acute uncomplicated cystitis (AUC) in adult women infected with susceptible isolates of E. coli and Enterococcus faecalis. We reviewed the literature regarding the use of oral fosfomycin for the treatment of AUC. All English-language references from 1975 to October 2015 were reviewed. In Canada, fosfomycin tromethamine is manufactured as Monurol $^{\circledR}$ and is available as a 3-gram single dose sachet. Fosfomycin has a unique chemical structure, inhibiting peptidoglycan synthesis at an earlier site compared to $\beta$-lactams with no cross-resistance with other agents. Fosfomycin displays broad-spectrum activity against ESBL-producing, AmpC-producing, carbapenem-non-susceptible, and multidrug-resistant (MDR) E. coli. Resistance to fosfomycin in E. coli is rare $(<1 \%)$. Fosfomycin is excreted unchanged in the urine by glomerular filtration with peak urinary concentration $\sim 4000 \mu \mathrm{g} / \mathrm{mL}$ and remains at concentrations $>100 \mu \mathrm{g} / \mathrm{mL}$ for 48 hours after a single 3-gram oral dose. No dosage adjustments are required in elderly patients, in pregnant patients, or in either renal or hepatic impairment. Fosfomycin demonstrates a favorable safety profile, and clinical trials have demonstrated efficacy in AUC that is comparable to ciprofloxacin, nitrofurantoin, and trimethoprim-sulfamethoxazole. Fosfomycin's in vitro activity against common uropathogens, including MDR isolates, its favorable safety profile including pregnancy patients, drug interactions, and clinical trials data demonstrating efficacy in AUC, has resulted in Canadian, US, and European guidelines/authorities recommending fosfomycin as a first line agent for the treatment of AUC.
\end{abstract}

\section{Introduction}

Urinary tract infections (UTIs) are among the most commonly occurring human infections $[1,2]$. It is estimated that approximately $50 \%$ of women will experience at least one UTI during their lifetime and that $25 \%$ will suffer recurrent infection [3]. Uncomplicated cystitis, the most common presentation for UTI, occurs in adult, premenopausal women with a normal, unobstructed, genitourinary tract where symptoms are confined to the urinary bladder and urethra. Females with cystitis typically present with dysuria and increased urinary urgency and frequency, as well as suprapubic pain, hematuria, and nocturia. Pyelonephritis is distinct from cystitis and is commonly associated with fever $\left(>38^{\circ} \mathrm{C}\right)$ and flank pain. The majority of cases of community-acquired cystitis are attributable to uropathogenic Escherichia coli (75-90\%) and Staphylococcus saprophyticus (5-15\%), with Klebsiella spp.,
Enterococcus spp., Streptococcus agalactiae, and Proteus mirabilis accounting for most other cases [1, 2, 4, 5]. Pseudomonas aeruginosa, Staphylococcus aureus, and Candida spp. are infrequent causes of acute uncomplicated UTIs.

Current guidelines published by the Infectious Diseases Society of America (IDSA) and the European Society for Clinical Microbiology and Infectious Diseases (ESCMID) recommend fosfomycin, nitrofurantoin, and trimethoprimsulfamethoxazole (TMP-SMX) as first-line agents to treat acute uncomplicated UTIs in adult females, reserving fluoroquinolones, amoxicillin-clavulanate, and other $\beta$-lactams as second-line agents [6]. Elevated rates of resistance ( $>10-20 \%)$ to TMP-SMX, as well as fluoroquinolones, are now widely reported for uropathogenic isolates of $E$. coli in Canada and elsewhere $[7,8]$. The most recently published Canadian study, describing antimicrobial resistance rates among E. coli isolated from patients with urinary tract infections, reported 
on isolates collected from 2010 to 2013 and found susceptibility rates of $74.7 \%$ to TMP-SMX, $77.4 \%$ to ciprofloxacin, $81.3 \%$ to amoxicillin-clavulanate, $96.1 \%$ to nitrofurantoin, and $99.4 \%$ to fosfomycin [9]. The increasing identification of extended-spectrum beta-lactamase- (ESBL-) producing E. coli across Canada and internationally has been associated with concomitant resistance to amoxicillin-clavulanate, ciprofloxacin, and TMP-SMX [7, 8]. Rates of susceptibility among $E$. coli of $<80 \%$ for one or more first- or second-line agents should prompt local reevaluation of empiric treatment strategies for acute uncomplicated UTIs [6].

This review endeavoured to summarize peer-reviewed published data on the development of fosfomycin, its chemistry, mechanisms of action and resistance, in vitro microbiology, pharmacokinetic and pharmacodynamic properties, efficacy demonstrated in clinical trials for acute cystitis, adverse effects, drug interactions, and its role in therapy of acute cystitis.

\section{Development of Fosfomycin}

In 1969, laboratories at MSD (Merck, Sharp, and Dohme) and CEPA (Compañia Española de Penicilina y Antibioticos) were the first to successfully isolate fosfomycin, a phosphonic acid derivative, from cultures of Streptomyces spp. (S. fradiae, S. viridochromogenes, and S. wedomorensis) [10, 11]. Fosfomycin was initially synthesized as a calcium salt [11]; however, this formulation was later identified to be responsible for discrepancies reported by in vitro studies and for delayed verification of therapeutic effectiveness in animal models and patients, and its commercial production was discontinued. Fosfomycin was subsequently reformulated into a new salt, fosfomycin tromethamine, also known as fosfomycin trometamol. Fosfomycin tromethamine demonstrated the same spectrum of activity and safety profile as the calcium salt as well as producing improved oral bioavailability and consistency for in vitro antimicrobial susceptibility testing $[12,13]$.

Fosfomycin has been available to physicians in many European countries as well as Japan, South Africa, and Brazil, in both oral and parenteral formulations, for up to four decades $[12,13]$. Parenteral fosfomycin is formulated as a disodium salt. Oral fosfomycin first entered the Canadian and American markets in 1997 [3] but was withdrawn in Canada several years later due to lack of use. It was recently reintroduced in Canada and has been added to several provincial formularies with an indication for the treatment of acute uncomplicated cystitis in adult women infected with susceptible isolates of E. coli and Enterococcus faecalis [14]. In Canada and the United States, fosfomycin tromethamine is manufactured exclusively under the brand name Monurol and is available as a 5.7-gram powder sachet of which 3 grams is fosfomycin [14].

\section{Chemistry}

The chemical structure of fosfomycin ([-] [1R, 2S]1,2-eposipropylphosphonic acid or cis-1,2-epoxypropyl

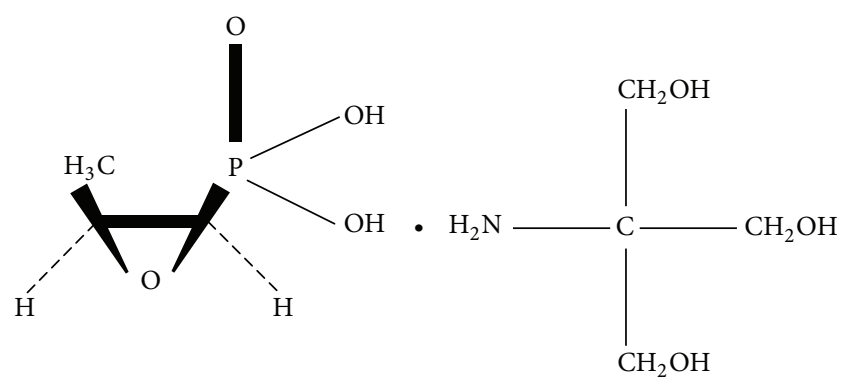

FIGURE 1: Chemical structure of fosfomycin tromethamine.

phosphoric acid) was published in 1969 shortly after its initial isolation [10] (Figure 1). It has a molecular mass of $138.059 \mathrm{~g} / \mathrm{mol}\left(\mathrm{C}_{3} \mathrm{H}_{7} \mathrm{O}_{4} \mathrm{P}\right)$. The structure of fosfomycin has two distinguishing features, a stable epoxide group and a phosphonic acid moiety; both are key components of its therapeutic activity. Its carbon-phosphorous bond is also unique to a minority of naturally occurring compounds and is an indication that it is the product of a distinct and complex biosynthetic process. The biosynthetic pathway of fosfomycin was initially described in $S$. wedomorensis and leads to the development of an in vitro chemical synthesis process, using phosphonic acid as starting material, which is currently used in commercial production [15].

\section{Mechanism of Action}

Fosfomycin's mechanism of action results from its irreversible inhibition of MurA (UDP-N-acetylglucosamine-3enolpyruvyl transferase), the cytosolic enzyme responsible for the first step in the peptidoglycan biosynthesis pathway that produces UDP-N-acetylmuramic acid [16]. More specifically, fosfomycin is a phosphoenolpyruvate (PEP) analog. MurA is responsible for ligating PEP to the $3^{\prime}$-hydroxyl group of UDP-N-acetylglucosamine in the pathway that produces UDP-N-acetylmuramic acid [16]. The inhibition of MurA is the result of direct nucleophilic attack by a catalytic site cysteine residue on the $\mathrm{C}-2$ carbon of fosfomycin, resulting in blockage of the catalytic site [16]. Fosfomycin has minimal to no effect on other enzymes utilizing PEP, such as enolase, pyruvate kinase, and PEP carboxykinase [16]. Fosfomycin's mechanism of action is unique and distinct from other bacterial cell wall inhibitors ( $\beta$-lactams and glycopeptides) as well as other classes of antibacterial agents suggesting that the likelihood of cross-resistance to these other agents should be minimal.

Fosfomycin enters the bacterial cytosol by two transport systems, the constitutively expressed L- $\alpha$-glycerophosphate (or glycerol-3-phosphate) uptake (GlpT) system and the glucose-6-phosphate- (G6P-) inducible hexose-monophosphate transport (UhpT) system. Of the two systems, the G6Pinducible UhpT system serves as the primary portal of entry for fosfomycin [16]. In 1983, Andrews and coworkers [17] demonstrated that a G6P concentration of $25 \mu \mathrm{g} / \mathrm{mL}$ optimizes induction of the UhpT system and it was subsequently added to standardized in vitro susceptibility testing methods 
for fosfomycin to facilitate reproducible MIC generation [18]. Most Enterobacteriaceae (excluding Proteus spp.), Enterococcus spp., and Staphylococcus spp. possess the UhpT transport system in their cell membrane [16, 17].

\section{Mechanisms of Resistance}

In vitro resistance to fosfomycin has been associated most commonly with chromosomal mutations in GlpT and less frequently with mutations in UhpT $[16,19-22]$. However, the presence of a functional G6P-inducible UhpT transport system frequently overrides resistance produced by mutations in GlpT and results in a fosfomycin susceptible phenotype [16]. Resistance to fosfomycin may also result less commonly from a myriad of other mechanisms including modification, inactivation, or overexpression of MurA, fosfomycin kinases, or the inactivation via plasmid-mediated enzymes such as fos $A$, fos $B$, fos $C$, and fos $X$ [23].

Plasmid-mediated fos enzymes are members of the glyoxalase superfamily and inactivate fosfomycin by catalyzing its conjugation with glutathione or another nucleophile. These enzymes function by nucleophilic attack on carbon 1 of fosfomycin, which opens the epoxide ring and inactivates it. The fos enzymes differ by the identity of the nucleophile utilized in the reaction: glutathione is used by FosA, bacillithiol by FosB, and water by FosX [21, 24, 25]. In general, FosA and FosX enzymes are produced by Gram-negative bacteria and FosB is produced by Gram-positive bacteria [21]. Another enzyme, FosC, utilizes ATP and adds a phosphate group to fosfomycin, which also neutralizes its antibacterial properties [26].

Resistance development during therapy is a confounding issue for fosfomycin. In vitro studies have shown that fosfomycin can be associated with the development of resistance at a frequency of $10^{-8}$ to $10^{-6}[23,27-29]$. However, the frequency of mutational resistance in vitro is not observed in clinical studies suggesting that there may be a biological cost associated with common mutations that confer resistance to fosfomycin in vitro $[22,23]$. Experimental studies with fosfomycin-resistant E. coli isolates have also demonstrated reduced epithelial cell adherence, increased susceptibility to polymorphonuclear cell and serum complement killing, and slower growth rates $[12,22,30]$. Overall, declines in bacterial virulence associated with fosfomycin-resistant $E$. coli may explain the low rates of resistance observed in vivo for this agent despite decades of use [12]. Data from the Antimicrobial Resistance Epidemiological Survey on Cystitis study showed that resistance to fosfomycin remains rare in regions where it is widely used ( 2\%) [31]. Other reasons for fosfomycin's low resistance rate in urinary tract infections may include its short contact time, high urine concentration, and potentially higher compliance compared with agents dosed for 3-7 days. Lower frequencies of resistance development have been observed at higher fosfomycin concentrations and in media with an acidic $\mathrm{pH}$ [23]. In vitro resistance development in $E$. coli is less frequent than in $K$. pneumoniae and $P$. aeruginosa [23]. Although the limited resistance in E. coli to fosfomycin from a variety of regions across the world (despite intensive use of this agent) is encouraging, we need to remain vigilant with ongoing surveillance studies to assess susceptibility to fosfomycin in Canada. Most worrisome would be the emergence of plasmid-mediated enzymes such as fos $A$, fos $B$, fos $C$, and fos $X$ [23].

\section{In Vitro Microbiology}

Standardized methods for antimicrobial susceptibility testing of fosfomycin are published by the Clinical and Laboratory Standards Institute (CLSI) [18] and the European Committee on Antimicrobial Susceptibility Testing (EUCAST) [32]. It is important to recognize that differences exist between these two standards. Specifically, there are differences in the organisms for which fosfomycin MIC and zone diameter breakpoints apply in the two standards as well as numerical differences in fosfomycin MIC and zone diameter breakpoints.

Currently, CLSI-approved agar dilution susceptibility breakpoints for fosfomycin exist only for $E$. coli and $E$. faecalis, with a MIC $\leq 64 \mu \mathrm{g} / \mathrm{mL}$ considered susceptible (resistance, $\geq 256 \mu \mathrm{g} / \mathrm{mL}$ ), and are approved only for testing isolates from urinary tract infections [18]. EUCAST breakpoints for fosfomycin apply to all Enterobacteriaceae with a $\mathrm{MIC} \leq 32 \mu \mathrm{g} / \mathrm{mL}$ considered susceptible (resistance, $>32 \mu \mathrm{g} / \mathrm{mL}$ ) for both oral (uncomplicated urinary tract infection only) and parenteral (systemic infections) fosfomycin [32]. EUCAST also publishes parenteral fosfomycin breakpoints for staphylococci but no breakpoints for enterococci. Based on our own data with E. coli, the difference in fosfomycin susceptibility using CLSI or EUCAST breakpoints is $<1 \%$ [9].

Antimicrobial susceptibility testing (agar dilution or disk diffusion) requires agar supplementation with $25 \mu \mathrm{g} / \mathrm{mL}$ of G6P to ensure induction of the UhpT pathway [18, 32]. Studies reporting in vitro susceptibility testing of fosfomycin prior to 1983 should be disregarded as the importance of adding physiological levels of G6P to testing media was unknown before that time $[12,17]$. Further, studies based upon broth dilution MIC testing should not be considered because of the relatively high likelihood of spontaneous mutation to fosfomycin resistance in broth [12]. Presently, we are not aware of efforts by automated susceptibility systems such as Vitek in testing fosfomycin in Canada or the US, but these efforts are required.

In general, fosfomycin demonstrates moderate in vitro potency against both Gram-negative and Gram-positive bacterial pathogens, including those most commonly associated with cystitis (E. coli, Klebsiella spp., Enterococcus spp., and Proteus spp., but not $S$. saprophyticus) [9, 12, 32-42].

Table 1 presents the in vitro activity of fosfomycin against aerobic and facultative Gram-negative bacteria from various specimen sources, including urine isolates. In all studies, MICs were determined using either the CLSI [18] or EUCAST [32] standard method. Fosfomycin has higher MICs for Klebsiella spp., Enterobacter spp., and Serratia spp., than for E. coli, Citrobacter spp., and Proteus spp. The activity of fosfomycin against Klebsiella spp. and Enterobacter spp. demonstrates some variability. Fosfomycin may be active against some isolates of $P$. aeruginosa with MICs ranging from 4 to $>512 \mu \mathrm{g} / \mathrm{mL}$. Acinetobacter baumannii appear inherently 
TABLE 1: In vitro activity of fosfomycin against aerobic and facultative Gram-negative bacteria ${ }^{a}$.

\begin{tabular}{|c|c|c|c|c|}
\hline \multirow{2}{*}{ Bacteria } & \multirow{2}{*}{ Number tested } & \multicolumn{3}{|c|}{ Fosfomycin } \\
\hline & & $\mathrm{MIC}_{50}(\mu \mathrm{g} / \mathrm{mL})$ & $\mathrm{MIC}_{90}(\mu \mathrm{g} / \mathrm{mL})$ & Range $(\mu \mathrm{g} / \mathrm{mL})$ \\
\hline Acinetobacter spp. & 185 & $64-128$ & $128-512$ & $0.25-512$ \\
\hline Citrobacter spp. (C. diversus and C. freundii) & 251 & $1-2$ & $4-16$ & $0.03-128$ \\
\hline Enterobacter spp. (E. agglomerans, E. aerogenes, and E. cloacae) & 779 & $8-64$ & $32-256$ & $0.12->512$ \\
\hline Escherichia coli & 9338 & $0.5-4$ & $2-16$ & $0.03-512$ \\
\hline Escherichia coli ESBL-producing & 362 & $0.5-2$ & $2-32$ & $0.03-512$ \\
\hline Escherichia coli AmpC-producing & 135 & 2 & $4-16$ & $\leq 1->512$ \\
\hline Haemophilus influenzae & 50 & 1 & 4 & $1-128$ \\
\hline Klebsiella oxytoca & 51 & 8 & 32 & $4-64$ \\
\hline Klebsiella pneumoniae & 392 & $16-32$ & $32->128$ & $0.5-512$ \\
\hline Klebsiella pneumoniae ESBL-producing & 74 & 16 & 64 & $2-256$ \\
\hline Klebsiella spp. & 995 & $8-16$ & $32-128$ & $\leq 2-512$ \\
\hline Morganella morganii & 98 & $128-256$ & 512 & $8->512$ \\
\hline Proteus mirabilis & 1472 & $\leq 2-4$ & $32->128$ & $\leq 1->512$ \\
\hline Proteus vulgaris (indole-positive Proteus) & 341 & $\leq 2-16$ & $8-256$ & $\leq 2-256$ \\
\hline Providencia spp. (P. rettgeri and P. stuartii) & 164 & $2-16$ & $8-128$ & $\leq 2-512$ \\
\hline Pseudomonas aeruginosa & 1518 & $32-256$ & $64-256$ & $4->512$ \\
\hline Pseudomonas spp. & 35 & 128 & 256 & $\leq 0.5-512$ \\
\hline Serratia marcescens & 307 & $8-16$ & $16-128$ & $\leq 2-128$ \\
\hline Shigella spp. & 185 & 2 & 2 & $0.5-64$ \\
\hline Stenotrophomonas maltophilia & 49 & 64 & 128 & $16-512$ \\
\hline
\end{tabular}

${ }^{a}$ Data compiled from $[9,12,32-41]$.

AmpC, chromosomal AmpC $\beta$-lactamase; ESBL, extended-spectrum $\beta$-lactamase.

$\mathrm{MIC}_{50}$, minimum concentration $(\mu \mathrm{g} / \mathrm{mL})$ required to inhibit the growth of $50 \%$ of isolates; $\mathrm{MIC}_{90}$, minimum concentration $(\mu \mathrm{g} / \mathrm{mL})$ required to inhibit the growth of $90 \%$ of isolates.

resistant to fosfomycin. However, studies combining fosfomycin with other agents such as cefepime and meropenem versus $A$. baumannii or an aminoglycoside for $P$. aeruginosa have demonstrated additivity between the two agents [43]. Gram-negative anaerobic bacteria have been reported to not be a part of fosfomycin's antibacterial spectrum [12] but the reasons for this are cryptic.

Fosfomycin retains its in vitro activity against ESBLproducing, AmpC-producing, carbapenem-non-susceptible, and multidrug-resistant (MDR) E. coli, as well as KPCproducing K. pneumoniae $[9,38-41,44]$. Kaase et al. tested 80 isolates of Enterobacteriaceae with various carbapenemases (KPC, VIM, NDM, and OXA-48) and reported that 78\% had MICs $\leq 32 \mu \mathrm{g} / \mathrm{mL}$ and would thus be considered susceptible according to the EUCAST breakpoint [45]. In another study, Falagas and others tested 152 MDR Enterobacteriaceae (76\% K. pneumoniae, $17 \%$ E. coli, 5\% P. mirabilis, and 2\% others) and determined that $93 \%$ of isolates were susceptible to fosfomycin by CLSI breakpoints (MIC, $\leq 64 \mu \mathrm{g} / \mathrm{mL}$ ) [46]. Subgroup analysis in the Falagas et al. study showed that $95 \%$, $94 \%$, and $83 \%$ of carbapenemase-producing $(n=79)$, ESBLproducing $(n=34)$, and metallo- $\beta$-lactamase-producing $(n=$ $24)$ isolates were susceptible to fosfomycin [46].

Table 2 summarizes data presented in the most recently published Canada-wide surveillance study of E. coli isolates collected from patients with UTIs from 2010 to 2013 [9]. Rates of susceptibility to fosfomycin were $99.4 \%, 97.9 \%, 99.1 \%$, $100 \%, 100 \%$, and $100 \%$ for all isolates, ciprofloxacin-resistant, TMP-SMX-resistant, ESBL-producing, AmpC-producing, and MDR isolates, respectively, superior to nitrofurantoin and other frequently prescribed oral empiric agents [9].

The in vitro activity of fosfomycin against Gram-positive bacteria from various specimen sources, including urine isolates, is summarized in Table 3. Fosfomycin appears more active in vitro against $S$. aureus, including methicillinresistant S. aureus (MRSA), and S. pneumoniae than other Gram-positive bacteria. The majority $(>50 \%)$ of isolates of $S$. aureus, enterococci (including vancomycin-resistant enterococci (VRE)), and streptococci have fosfomycin MICs $\leq 32 \mu \mathrm{g} / \mathrm{mL}$. Some streptococci, Staphylococcus saprophyticus, corynebacteria, Chlamydia, and mycoplasmas are resistant to fosfomycin likely due to the absence or low abundance of the G6P-inducible UhpT system or an altered MurA target $[12,47]$.

\section{Pharmacokinetic Properties}

Fosfomycin is freely soluble in water, demonstrates negligible plasma protein binding, and distributes widely into tissues 
TABLE 2: In vitro activities of orally prescribed antimicrobial agents against E. coli isolated from urine specimens in clinical laboratories across Canada from 2010 to $2013^{\mathrm{a}}$.

\begin{tabular}{|c|c|c|c|}
\hline \multirow{2}{*}{ E. coli isolate phenotype $\mathrm{e}^{\mathrm{b}}$ (number tested) } & \multirow{2}{*}{ Antimicrobial agent } & \multicolumn{2}{|c|}{ MIC interpretation } \\
\hline & & $\%$ susceptible & $\%$ resistant \\
\hline \multirow{5}{*}{ All E. coli (868) } & Fosfomycin & 99.4 & 0.1 \\
\hline & Amoxicillin-clavulanate & 81.3 & 5.7 \\
\hline & Ciprofloxacin & 77.4 & 22.5 \\
\hline & Nitrofurantoin & 96.1 & 1.5 \\
\hline & TMP-SMX ${ }^{c}$ & 74.7 & 25.3 \\
\hline \multirow{5}{*}{ TMP-SMX-resistant (219) } & Fosfomycin & 99.1 & 0 \\
\hline & Amoxicillin-clavulanate & 67.1 & 6.4 \\
\hline & Ciprofloxacin & 51.6 & 47.9 \\
\hline & Nitrofurantoin & 91.8 & 3.2 \\
\hline & TMP-SMX & 0 & 100 \\
\hline \multirow{5}{*}{ Ciprofloxacin-resistant (195) } & Fosfomycin & 97.9 & 0 \\
\hline & Amoxicillin-clavulanate & 66.0 & 6.7 \\
\hline & Ciprofloxacin & 0 & 100 \\
\hline & Nitrofurantoin & 91.3 & 4.1 \\
\hline & TMP-SMX & 45.9 & 54.1 \\
\hline \multirow{5}{*}{ ESBL-producing (42) } & Fosfomycin & 100 & 0 \\
\hline & Amoxicillin-clavulanate & 33.3 & 11.9 \\
\hline & Ciprofloxacin & 9.5 & 90.5 \\
\hline & Nitrofurantoin & 83.3 & 4.8 \\
\hline & TMP-SMX & 35.7 & 64.3 \\
\hline \multirow{5}{*}{ AmpC-producing (16) } & Fosfomycin & 100 & 0 \\
\hline & Amoxicillin-clavulanate & 6.3 & 87.4 \\
\hline & Ciprofloxacin & 75.0 & 25.0 \\
\hline & Nitrofurantoin & 100 & 0 \\
\hline & TMP-SMX & 75.0 & 25.0 \\
\hline \multirow{5}{*}{ Multidrug-resistant (15) } & Fosfomycin & 100 & 0 \\
\hline & Amoxicillin-clavulanate & 13.3 & 66.7 \\
\hline & Ciprofloxacin & 0 & 100 \\
\hline & Nitrofurantoin & 60.0 & 40.0 \\
\hline & TMP-SMX & 6.7 & 93.3 \\
\hline
\end{tabular}

${ }^{\mathrm{a}}$ Data adapted from [9].

${ }^{\mathrm{b}}$ ESBL, extended-spectrum $\beta$-lactamase; AmpC, chromosomal AmpC $\beta$-lactamase; multidrug-resistant was defined as isolates resistant to $\geq 3$ agents from different antimicrobial classes (amoxicillin-clavulanate, ciprofloxacin, nitrofurantoin, and TMP-SMX).

${ }^{\mathrm{c}}$ TMP-SMX, trimethoprim-sulfamethoxazole.

TABLE 3: In vitro activity of fosfomycin against facultative Gram-positive bacteria ${ }^{\mathrm{a}}$.

\begin{tabular}{|c|c|c|c|c|}
\hline \multirow{2}{*}{ Organism } & \multirow{2}{*}{ Number tested } & \multicolumn{3}{|c|}{ Fosfomycin } \\
\hline & & $\mathrm{MIC}_{50}(\mu \mathrm{g} / \mathrm{mL})$ & $\mathrm{MIC}_{90}(\mu \mathrm{g} / \mathrm{mL})$ & Range $(\mu \mathrm{g} / \mathrm{mL})$ \\
\hline Enterococcus faecalis & 1862 & 32 & 64 & $0.5-512$ \\
\hline Enterococcus faecium & 516 & $32-64$ & $64-128$ & $0.5-128$ \\
\hline Enterococcus spp. & 137 & $16-32$ & 64 & $0.25->256$ \\
\hline Staphylococcus aureus & 2213 & 4 & 16 & $0.12-512$ \\
\hline Staphylococcus aureus, MRSA & 162 & 4 & 64 & $0.5-512$ \\
\hline Staphylococcus epidermidis & 896 & 8 & 128 & $0.5-256$ \\
\hline Staphylococcus saprophyticus & 227 & $64-128$ & $256->512$ & $\leq 2->512$ \\
\hline Streptococcus pneumoniae & 57 & 8 & 16 & $4-32$ \\
\hline Streptococcus pyogenes & 50 & 32 & 64 & $2-64$ \\
\hline Streptococcus agalactiae & 50 & 32 & 64 & $2-64$ \\
\hline
\end{tabular}

${ }^{\mathrm{a}}$ Data compiled from $[12,32-34,38,42]$. 
TABLE 4: Pharmacokinetic properties of fosfomycin following a single 3-gram oral dose $\mathrm{e}^{\mathrm{a}}$.

\begin{tabular}{lc}
\hline Parameter & Mean value or range \\
\hline Serum/plasma & $34-41 \%$ \\
Bioavailability $(F)$ & $22-32 \mu \mathrm{g} / \mathrm{mL}$ \\
Maximum plasma concentration $C_{\max }$ & $2-2.5 \mathrm{~h}$ \\
Time to maximum concentration in the blood $\left(T_{\max }\right)$ & $145 \mathrm{mg} / \mathrm{L} \cdot \mathrm{h}$ \\
Area under the curve $(\mathrm{AUC})$ & $136.1 \mathrm{~L}$ \\
Volume of distribution $\left(V_{d}\right)$ & $5.7 \mathrm{~h}$ \\
Half-life $\left(t_{1 / 2}\right)$ & $16.9 \mathrm{~L} / \mathrm{h}$ \\
Clearance $(\mathrm{CL})$ & $1053-4415 \mu \mathrm{g} / \mathrm{mL}$ \\
\hline Urine & $4 \mathrm{~h}$ \\
Maximum urinary concentration $\left(U_{\max }\right)$ & $\sim 100 \mu \mathrm{gg} / \mathrm{mL}$ \\
Time to maximum concentration in the urine $\left(\right.$ urinary $\left.t_{\max }\right)$ & None required \\
Urinary concentration at $48 \mathrm{~h}$ & None required \\
\hline Dosage adjustments & None required \\
Dose adjustment in elderly & None required \\
Dose adjustment in pregnancy & \\
Dose adjustment for renal impairment &
\end{tabular}

(steady state volume of distribution is $136.1 \pm 44.1 \mathrm{~L}$ ) [12, 14, 23, 27, 43, 48-50].

Table 4 summarizes the pharmacokinetic properties for fosfomycin tromethamine following oral administration of a single 5.7-gram (3-gram fosfomycin) dose. The oral bioavailability of fosfomycin is $34-41 \%, 18 \%$ is recovered from feces, and $54-65 \%$ of absorbed fosfomycin is recovered unaltered in the urine $[14,48]$.

Fosfomycin's distribution follows a two-compartment model $[48,51]$. Upon absorption from the gut, fosfomycin is rapidly distributed to the kidneys, bladder, prostate, and seminal vesicles $[14,49]$. A 3-gram oral dose of fosfomycin results in serum $C_{\max }$ of $22-32 \mu \mathrm{g} / \mathrm{mL}$ achieved within 22.5 hours. Animal studies have demonstrated that fosfomycin penetrates fluids and tissue of the central nervous system and cardiac, respiratory, and urinary systems supporting its use in noncystitis indications such as prostatitis [52].

Fosfomycin is not metabolized but rather is excreted unchanged in the urine by glomerular filtration [14, 49]. Its serum half-life is 5.7 hours $[12,14]$. Peak urinary concentrations reach $\sim 4000 \mu \mathrm{g} / \mathrm{mL}$ and remain at concentrations $>100 \mu \mathrm{g} / \mathrm{mL}$ for 48 hours [49]. A fosfomycin urinary concentration above the $\mathrm{MIC}_{90}$ of $E$. coli $(4 \mu \mathrm{g} / \mathrm{mL})$ has been reported for 80 hours [48].

No dosage adjustments are required in elderly patients, pregnant patients, or either renal or hepatic impairment $[14,48,49]$. Studies have reported an increase in $T_{\max }$ and $C_{\max }$, larger $\mathrm{AUC}$, and reduced rates of elimination for renally impaired patients; regardless, no dosage adjustment is recommended for patients with a creatinine clearance $\left(\mathrm{Cr}_{\mathrm{CL}}\right)>10 \mathrm{~mL} / \mathrm{min}[14,48,49]$. A study of patients with $\left(\mathrm{Cr}_{\mathrm{CL}}\right)$ of $7-54 \mathrm{~mL} / \mathrm{min}$ demonstrated increased serum $t_{1 / 2}$ from $11 \mathrm{~h}$ to $50 \mathrm{~h}$, accompanied by the observation that only $30 \%$ of orally absorbed fosfomycin was excreted within
24 hours [49]. Another study of patients with mean $\mathrm{Cr}_{\mathrm{CL}}$ of $40 \mathrm{~mL} / \mathrm{min}$ demonstrated lower mean urinary concentrations for the first 24 hours, but similar concentrations after 48-60 hours [14]. Limited data exists for both children and pregnant women. Studies have demonstrated slightly higher elimination rates in children, as well as an increase in mean urinary fosfomycin concentrations in pregnant patients, although the differences were considered insignificant and did not warrant dose adjustments [14].

\section{Pharmacodynamic Properties}

Fosfomycin has demonstrated concentration-dependent killing in two different in vitro models. Mazzei and colleagues assessed fosfomycin activity using kill curves at concentrations from $1 \mathrm{x}$ MIC to $64 \mathrm{x}$ MIC for isolates of E. coli and P. mirabilis [53]. When bacterial growth was assessed from time 0 to 24 hours, bacterial inhibition was directly proportional to fosfomycin concentration [53]. For E. coli, complete eradication was observed at 6-8 hours at fosfomycin concentrations $\geq 4 \mathrm{x}$ MIC [53].

In a hollow fiber infection model that simulated human pharmacokinetics of fosfomycin, three isolates of ESBLproducing E. coli (Ec2974: fosfomycin MICs $1 \mu \mathrm{g} / \mathrm{mL}$, Ec46: MIC $1 \mu \mathrm{g} / \mathrm{mL}$, and Ec4244: MIC $64 \mu \mathrm{g} / \mathrm{mL}$, resp.) were challenged with varying dosage regimens to assess bacterial inhibition, including $4 \mathrm{~g}$ q8h (12 g/day), $8 \mathrm{~g}$ q8h ( $24 \mathrm{~g} /$ day), and $12 \mathrm{~g} \mathrm{q} 8 \mathrm{~h}$ (36g/day), and concentration-dependent activity was observed [54]. Fosfomycin $f \mathrm{AUC}_{0-24} / \mathrm{MIC}$ ratios $(\mathrm{MIC}$, $1 \mu \mathrm{g} / \mathrm{mL}$ ) were determined to be $1,744.94,3,3136.03$, and 4,287.82 for $12 \mathrm{~g} /$ day, $24 \mathrm{~g} /$ day, and $36 \mathrm{~g} /$ day doses, respectively [54]. While growth of highly resistant mutants in the $12 \mathrm{~g} / \mathrm{day}$ dose was observed after 24 hours, higher dosages ( $24 \mathrm{~g}$ /day and $36 \mathrm{~g} /$ day) resulted in complete bacterial eradication 
and suppression of resistant mutants [54]. Furthermore, no difference in the rate and extent of fosfomycin's bactericidal activity was observed with different dosage intervals suggesting that fosfomycin's pharmacodynamic activity was not time-dependent [54].

The postantibiotic effect (PAE) for fosfomycin has also been assessed [53]. Following two-hour exposures to varying concentrations of fosfomycin (0.25x MIC, 1x MIC, 4x MIC, and $8 \mathrm{x} \mathrm{MIC}$ ) and subsequent removal, E. coli and P. mirabilis demonstrated long, concentration-dependent PAEs ranging from 3.4 hours at $0.25 \mathrm{x}$ MIC to 3.4 hours at $1 \mathrm{x}$ MIC to $4.2 \mathrm{~h}$ at $8 \mathrm{x}$ MIC $[49,53]$.

\section{Clinical Trials}

In 2010, Falagas and others published a meta-analysis of all available, randomized controlled trials comparing fosfomycin and other antimicrobial agents for effectiveness and safety in the treatment of acute uncomplicated cystitis [46]. Following screening, they included 27 trials from 1987 to January 2010 in their analysis [46]. They reported no difference in clinical success, microbiological success, relapse, or reinfection with single dose fosfomycin and comparators using multidose regimens in trials involving nonpregnant females ( $n=16$ trials) and trials involving adult mixed populations ( $n=3$ trials). They were unable to draw conclusions from trials involving pediatric patients ( $n=3$ trials) and pregnant patients ( $n=5$ trials) because of insufficient data. Fosfomycin was shown to possess a comparable safety profile with the comparators prescribed for nonpregnant women, adult mixed populations, and pediatric patients and to have a significantly lower rate of adverse events in pregnant women [46]. The authors concluded that fosfomycin may provide a valuable alternative therapy for the treatment of acute cystitis in nonpregnant and pregnant women, as well as in the elderly and pediatric patients.

To further the work of Falagas et al., we performed a review of randomized, controlled trials from 2010 to 2015 that compared fosfomycin and other antimicrobial agents for the treatment of acute uncomplicated cystitis and one additional study was identified. Ceran and colleagues conducted a randomized comparative study of single-dose fosfomycin and 5-day ciprofloxacin in 260 female patients, aged between 18 and 65 years, with uncomplicated lower urinary tract infections [55]. Of the 260 patients enrolled, 142 completed the study. In the 142 patients, E. coli $(82.3 \%)$ and Enterobacter spp. (8.4\%) were the pathogens most frequently isolated from urine cultures. Patients were evaluated on days 7-10 for clinical and microbiological cure. Clinical cure rates were $83 \%$ for fosfomycin and $81 \%$ for ciprofloxacin; microbiological cure rates were $83 \%$ for fosfomycin and $78 \%$ for ciprofloxacin. Patients were reevaluated on day 60 for recurrence or reinfection; only five patients of 142 patients demonstrated relapse. The authors concluded that a single 3-gram dose of fosfomycin was as effective as ciprofloxacin, at $500 \mathrm{mg}$ twice a day for 5 days, in the treatment of acute uncomplicated cystitis.
TABLE 5: Adverse effects of fosfomycin following a single 3-gram oral dose ${ }^{\mathrm{a}}$.

\begin{tabular}{lc}
\hline Adverse effect & Frequency $(\%)$ \\
\hline Diarrhea & $4-10$ \\
Vaginitis & 5.8 \\
Headache & 2 \\
Nausea & $2-5$ \\
Epigastric/abdominal pain & $1.3-2$ \\
Dyspepsia & $1-2$ \\
Dizziness & 1.2 \\
Asthenia & 1 \\
Fatigue & 0.2 \\
Mild backache & 0.2 \\
\hline
\end{tabular}

${ }^{\mathrm{a}}$ Data adapted from $[47,48,56]$.

\section{Adverse Effects}

Most adverse effects described for fosfomycin have been mild and transient [48]. Table 5 summarizes the most commonly reported adverse effects of fosfomycin. Serious adverse reactions such as angioedema, asthma exacerbation, cholestatic jaundice, hepatic necrosis, and toxic megacolon have been extremely rare [48]. As with other antimicrobial agents, prolonged use of fosfomycin may be associated with fungal or bacterial super-infections, including Clostridium difficile infection [47]. A postmarketing study in Japan (reporting on fosfomycin calcium) documented only 2 cases of super-infection within 6 years [56]. Laboratory changes (including increased eosinophil count, increased or decreased white blood cell count, increased bilirubin, increased alanine aminotransferase, increased aspartate aminotransferase, increased alkaline phosphatase, decreased hematocrit, decreased hemoglobin, and alterations in platelet count) have also been reported; however, they have been transient and clinically insignificant [47].

\section{Drug Interactions}

The only confirmed drug interaction for fosfomycin is with metoclopramide [14, 47, 49, 57]. Metoclopramide's mechanism of action (increase in gastrointestinal mobility) decreases the absorption of fosfomycin tromethamine, resulting in a $25 \%$ reduction in $C_{\max }$ and 20 -minute shorter $t_{\max }[47,48]$. Recent data also suggest that fosfomycin may have a protective effect against nephrotoxicity induced by aminoglycosides or cisplatin, which may be due to fosfomycin's inhibition of medication-induced histamine release from mast cells [56]. Lastly, no drug interactions have been reported between fosfomycin and commonly used agents such as analgesics, anticonvulsants, bronchodilators, diuretics, spasmolytics, and antipyretics [14].

\section{Role of Fosfomycin in Therapy of Acute Cystitis}

In vitro studies have demonstrated increasing rates of resistance among $E$. coli urinary isolates to trimethoprimsulfamethoxazole and ciprofloxacin, antimicrobial agents 
frequently prescribed for the treatment of cystitis $[7,57,58]$. Community-acquired UTIs caused by ESBL-producing $E$. coli are also being reported with increasing frequency [59]. These isolates are often MDR, leaving clinicians with few oral treatment options [59]. Fosfomycin demonstrates excellent in vitro activity against common uropathogens, including MDR isolates [9]. Fosfomycin demonstrates a favorable safety profile, and clinical trials have demonstrated efficacy in the treatment of uncomplicated cystitis that is comparable to other first-line antimicrobials $[46,48,60]$. Fosfomycin has a unique chemical structure that is distinct from all other marketed antimicrobial classes (i.e., $\beta$-lactams, glycopeptides, fluoroquinolones, macrolides, lincosamides, tetracyclines, aminoglycosides, etc.) and there is no cross-resistance with other agents used to treat cystitis [12]. Resistance to fosfomycin most commonly arises by chromosomal mutations which do not spread easily, and the biological cost of these genetic mutations is high $[22,23]$. As an additional advantage, when used for the treatment of acute uncomplicated cystitis, fosfomycin is conveniently administered as a single $3 \mathrm{~g}$ oral dose [6]. Current UTI guidelines from the Infectious Diseases Society of America, the European Society for Microbiology and Infectious Diseases, the European Association of Urology, and the Canadian Anti-Infective Guidelines for Community Acquired Infections (the Ontario Orange Book) all recommend fosfomycin as a first-line antimicrobial for the treatment of acute, uncomplicated cystitis [6, 60-62]. This recommendation is supported by the data reviewed here. Given the rising antimicrobial resistance rates among common uropathogens, it is likely that fosfomycin will be used with increasing frequency over the coming years.

\section{Competing Interests}

Drs. James A. Karlowsky and Andrew J. Walkty declare that they have no competing interests. Dr. George G. Zhanel declares receiving research funding from Paladin labs.

\section{Acknowledgments}

The authors would like to thank Camille Manansala for her contributions to the initial literature review and to the paper outline.

\section{References}

[1] A. Sultan, M. Rizvi, F. Khan, H. Sami, I. Shukla, and H. M. Khan, "Increasing antimicrobial resistance among uropathogens: is fosfomycin the answer?" Urology Annals, vol. 7, no. 1, pp. 2630, 2015.

[2] M. A. Pezzlo, "Laboratory diagnosis of urinary tract infections: guidelines, challenges, and innovations," Clinical Microbiology Newsletter, vol. 36, no. 12, pp. 87-93, 2014.

[3] S. A. Mehnert-Kay, "Diagnosis and management of uncomplicated urinary tract infections," American Family Physician, vol. 72, no. 3, pp. 451-458, 2005.

[4] A. Wang, P. Nizran, M. A. Malone, and T. Riley, "Urinary tract infections," Primary Care: Clinics in Office Practice, vol. 40, no. 3, pp. 687-706, 2013.
[5] L. Nicolle, P. A. M. Anderson, J. Conly et al., "Uncomplicated urinary tract infection in women. Current practice and the effect of antibiotic resistance on empiric treatment," Canadian Family Physician, vol. 52, pp. 612-618, 2006.

[6] K. Gupta, T. M. Hooton, K. G. Naber et al., "International clinical practice guidelines for the treatment of acute uncomplicated cystitis and pyelonephritis in women: A 2010 update by the Infectious Diseases Society of America and the European Society for Microbiology and Infectious Diseases," Clinical Infectious Diseases, vol. 52, no. 5, pp. e103-e120, 2011.

[7] J. A. Karlowsky, P. R. S. Lagacé-Wiens, P. J. Simner et al., "Antimicrobial resistance in urinary tract pathogens in Canada from 2007 to 2009: CANWARD surveillance study," Antimicrobial Agents and Chemotherapy, vol. 55, no. 7, pp. 3169-3175, 2011.

[8] A. Sorlozano, A. Jimenez-Pacheco, J. de Dios Luna del Castillo et al., "Evolution of the resistance to antibiotics of bacteria involved in urinary tract infections: a 7-year surveillance study," American Journal of Infection Control, vol. 42, no. 10, pp. 10331038, 2014.

[9] J. A. Karlowsky, A. J. Denisuik, P. R. S. Lagacé-Wiens et al., "In vitro activity of fosfomycin against Escherichia coli isolated from patients with urinary tract infections in Canada as part of the CANWARD surveillance study," Antimicrobial Agents and Chemotherapy, vol. 58, no. 2, pp. 1252-1256, 2014.

[10] B. G. Christensen, W. J. Leanza, T. R. Beattie et al., "Phosphonomycin: structure and synthesis," Science, vol. 166, pp. 123-125, 1969.

[11] G. G. Grassi, "Fosfomycin trometamol: historical background and clinical development," Infection, vol. 18, supplement 2, pp. S57-S59, 1990.

[12] M. Popovic, D. Steinort, S. Pillai, and C. Joukhadar, "Fosfomycin: an old, new friend?" European Journal of Clinical Microbiology and Infectious Diseases, vol. 29, no. 2, pp. 127-142, 2010.

[13] P. J. Bergen, C. B. Landersdorfer, H. J. Lee, J. Li, and R. L. Nation, "'Old' antibiotics for emerging multidrug-resistant bacteria," Current Opinion in Infectious Diseases, vol. 25, no. 6, pp. 626633, 2012.

[14] Monurol ${ }^{\circledR}$ Fosfomycin Trometamol Product Monograph, Springer Healthcare Italia Srl, Milano, Italia, 2012.

[15] R. D. Woodyer, Z. Shao, P. M. Thomas et al., "Heterologous production of fosfomycin and identification of the minimal biosynthetic gene cluster," Chemistry and Biology, vol. 13, no. 11, pp. 1171-1182, 2006.

[16] F. M. Kahan, J. S. Kahan, P. J. Cassidy, and H. Kropp, “The mechanism of action of fosfomycin (phosphonomycin)," Annals of the New York Academy of Sciences, vol. 235, pp. 364-386, 1974.

[17] J. M. Andrews, F. Baquero, J. M. Beltran et al., "International collaborative study on standardization of bacterial sensitivity to fosfomycin," Journal of Antimicrobial Chemotherapy, vol. 12, no. 4, pp. 357-361, 1983.

[18] Clinical and Laboratory Standards Institute, "Performance standards for antimicrobial susceptibility testing; Twenty-fifth informational supplement," Tech. Rep. M100-S25, 2015.

[19] P.-L. Ho, J. Chan, W.-U. Lo et al., "Prevalence and molecular epidemiology of plasmid-mediated fosfomycin resistance genes among blood and urinary Escherichia coli isolates," Journal of Medical Microbiology, vol. 62, no. 11, pp. 1707-1713, 2013.

[20] J. Navas, J. León, M. Arroyo, and J. M. García Lobo, "Nucleotide sequence and intracellular location of the product of the 
fosfomycin resistance gene from transposon Tn2921," Antimicrobial Agents and Chemotherapy, vol. 34, no. 10, pp. 2016-2018, 1990.

[21] R. E. Rigsby, K. L. Fillgrove, L. A. Beihoffer, and R. N. Armstrong, "Fosfomycin resistance proteins: a nexus of glutathione transferases and epoxide hydrolases in a metalloenzyme superfamily," Methods in Enzymology, vol. 401, pp. 367-379, 2005.

[22] M. Ballestero-Tellez, F. Docobo-Perez, J. M. RodriguezMartinez et al., "Contribution of specific resistance mechanisms of fosfomycin resistance and its biological cost in Escherichia coli," in Proceedings of the Interscience Conference on Antimicrobial Agents and Chemotherapy (ICAAC '15), September 2015.

[23] D. E. Karageorgopoulos, R. Wang, X.-H. Yu, and M. E. Falagas, "Fosfomycin: evaluation of the published evidence on the emergence of antimicrobial resistance in gram-negative pathogens," Journal of Antimicrobial Chemotherapy, vol. 67, no. 2, Article ID dkr466, pp. 255-268, 2012.

[24] S. V. Sharma, V. K. Jothivasan, G. L. Newton et al., "Chemical and chemoenzymatic syntheses of bacillithiol: a unique lowmolecular-weight thiol amongst low $\mathrm{G}+\mathrm{C}$ gram-positive bacteria," Angewandte Chemie-International Edition, vol. 50, no. 31, pp. 7101-7104, 2011.

[25] A. A. Roberts, S. V. Sharma, A. W. Strankman, S. R. Duran, M. Rawat, and C. J. Hamilton, "Mechanistic studies of FosB: a divalent-metal-dependent bacillithiol-S-transferase that mediates fosfomycin resistance in Staphylococcus aureus," Biochemical Journal, vol. 451, no. 1, pp. 69-79, 2013.

[26] P. García, P. Arca, and J. Evaristo Suárez, "Product of fos $C$, a gene from Pseudomonas syringae, mediates fosfomycin resistance by using ATP as cosubstrate," Antimicrobial Agents and Chemotherapy, vol. 39, no. 7, pp. 1569-1573, 1995.

[27] F. Gudiol, "S55 Facts and myths about fosfomycin," International Journal of Antimicrobial Agents, vol. 29, supplement 2, pp. S12S13, 2007.

[28] J. Oteo, V. Bautista, N. Lara et al., "Parellel increase in community use of fosfomycin and resistance to fosfomycin in extendedspectrum beta-lactamase-producing Escherichia coli," Journal of Antimicrobial Chemotherapy, vol. 65, pp. 2459-2463, 2010.

[29] J. Oteo, V. Bautista, N. Lara et al., "Parallel increase in community use of fosfomycin and resistance to fosfomycin in extended-spectrum $\beta$-lactamase (ESBL)-producing Escherichia coli, Journal of Antimicrobial Chemotherapy, vol. 65, no. 11, Article ID dkq346, pp. 2459-2463, 2010.

[30] A. Marchese, L. Gualco, E. A. Debbia, G. C. Schito, and A. M. Schito, "In vitro activity of fosfomycin against Gram-negative urinary pathogens and the biological cost of fosfomycin resistance," International Journal of Antimicrobial Agents, vol. 22, no. 2, pp. S53-S59, 2003.

[31] G. C. Schito, K. G. Naber, H. Botto et al., "The ARESC study: an international survey on the antimicrobial resistance of pathogens involved in uncomplicated urinary tract infections," International Journal of Antimicrobial Agents, vol. 34, no. 5, pp. 407-413, 2009.

[32] European Committee on Antimicrobial Susceptibility Testing Clinical breakpoints-bacteria, version 5.0. 2015.

[33] A. L. Barry and P. C. Fuchs, "In vitro susceptibility testing procedures for fosfomycin tromethamine," Antimicrobial Agents and Chemotherapy, vol. 35, no. 6, pp. 1235-1238, 1991.

[34] A. L. Barry and S. D. Brown, "Antibacterial spectrum of fosfomycin trometamol," Journal of Antimicrobial Chemotherapy, vol. 35, no. 1, pp. 228-230, 1995.
[35] L. V. Perdigão-Neto, M. S. Oliveira, C. F. Rizek, C. M. D. M. Carrilho, S. F. Costa, and A. S. Levin, "Susceptibility of multiresistant gram-negative bacteria to fosfomycin and performance of different susceptibility testing methods," Antimicrobial Agents and Chemotherapy, vol. 58, no. 3, pp. 1763-1767, 2014.

[36] A. Alhambra, J. A. Cuadros, J. Cacho, J. L. Gómez-Garcés, and J. I. Alós, "In vitro susceptibility of recent antibiotic-resistant urinary pathogens to ertapenem and 12 other antibiotics," Journal of Antimicrobial Chemotherapy, vol. 53, no. 6, pp. 1090-1094, 2004.

[37] S. Rajenderan, V. Balaji, S. Anandan et al., "Determination of MIC distribution of arbekacin, cefminox, fosfomycin, biapenem and other antibiotics against gram-negative clinical isolates in South India: a prospective study," PLOS ONE, vol. 9, article 7, Article ID e103253, 2014.

[38] H. Hayami, S. Takahashi, K. Ishikawa et al., "Nationwide surveillance of bacterial pathogens from patients with acute uncomplicated cystitis conducted by the Japanese surveillance committee during 2009 and 2010: antimicrobial susceptibility of Escherichia coli and Staphylococcus saprophyticus," Journal of Infection and Chemotherapy, vol. 19, no. 3, pp. 393-403, 2013.

[39] K. S. Ko, J. Y. Suh, K. R. Peck et al., "In vitro activity of fosfomycin against ciprofloxacin-resistant or extended-spectrum $\beta$-lactamase-producing Escherichia coli isolated from urine and blood," Diagnostic Microbiology and Infectious Disease, vol. 58, no. 1, pp. 111-115, 2007.

[40] M. Kresken, Y. Pfeifer, D. Hafner, R. Wresch, and B. KörberIrrgang, "Occurrence of multidrug resistance to oral antibiotics among Escherichia coli urine isolates from outpatient departments in Germany: extended-spectrum $\beta$-lactamases and the role of fosfomycin," International Journal of Antimicrobial Agents, vol. 44, no. 4, pp. 295-300, 2014.

[41] M.-S. Hsu, C.-H. Liao, C.-Y. Liu, C.-J. Yang, Y.-T. Huang, and P.-R. Hsueh, "In vitro susceptibilities of clinical isolates of ertapenem-non-susceptible Enterobacteriaceae to nemonoxacin, tigecycline, fosfomycin and other antimicrobial agents," International Journal of Antimicrobial Agents, vol. 37, no. 3, pp. 276-278, 2011.

[42] M. B. Perri, E. Hershberger, M. Ionescu, C. Lauter, and M. J. Zervos, "In vitro susceptibility of vancomycin-resistant enterococci (VRE) to fosfomycin," Diagnostic Microbiology and Infectious Disease, vol. 42, no. 4, pp. 269-271, 2002.

[43] M. E. Falagas, K. P. Giannopoulou, G. N. Kokolakis, and P. I. Rafailidis, "Fosfomycin: use beyond urinary tract and gastrointestinal infections," Clinical Infectious Diseases, vol. 46, no. 7, pp. 1069-1077, 2008.

[44] A. Endimiani, G. Patel, K. M. Hujer et al., "In vitro activity of fosfomycin against ${ }^{\text {bla }} \mathrm{KPC}$-containing Klebsiella pneumoniae isolates, including those nonsusceptible to tigecycline and/or colistin," Antimicrobial Agents and Chemotherapy, vol. 54, no. 1, pp. 526-529, 2010.

[45] M. Kaase, F. Szabados, A. Anders, and S. G. Gatermann, "Fosfomycin susceptibility in carbapenem-resistant Enterobacteriaceae from Germany," Journal of Clinical Microbiology, vol. 52, no. 6, pp. 1893-1897, 2014.

[46] M. E. Falagas, S. Maraki, D. E. Karageorgopoulos, A. C. Kastoris, E. Mavromanolakis, and G. Samonis, "Antimicrobial susceptibility of multidrug-resistant (MDR) and extensively drugresistant (XDR) Enterobacteriaceae isolates to fosfomycin," International Journal of Antimicrobial Agents, vol. 35, no. 3, pp. 240-243, 2010. 
[47] A. S. Michalopoulos, I. G. Livaditis, and V. Gougoutas, “The revival of fosfomycin," International Journal of Infectious Diseases, vol. 15, no. 11, pp. e732-e739, 2011.

[48] S. S. Patel, J. A. Balfour, and H. M. Bryson, "Fosfomycin Tromethamine. A review of its antibacterial activity, pharmacokinetic properties and therapeutic efficacy as a single-dose oral treatment for acute uncomplicated lower urinary tract infections," Drugs, vol. 53, no. 4, pp. 637-656, 1997.

[49] G. M. Keating, "Fosfomycin trometamol: a review of its use as a single-dose oral treatment for patients with acute lower urinary tract infections and pregnant women with asymptomatic bacteriuria," Drugs, vol. 73, no. 17, pp. 1951-1966, 2013.

[50] N. Roussos, D. E. Karageorgopoulos, G. Samonis, and M. E. Falagas, "Clinical significance of the pharmacokinetic and pharmacodynamics characteristics of fosfomycin for the treatment of patients with systemic infections," International Journal of Antimicrobial Agents, vol. 34, pp. 506-515, 2009.

[51] W. M. M. Kirby, "Pharmacokinetics of fosfomycin," Chemotherapy, vol. 23, pp. 141-151, 1977.

[52] M. E. Falagas and P. I. Rafailidis, "Fosfomycin: the current status of the drug," Clinical Infectious Diseases, vol. 61, no. 7, pp. 11441146, 2015.

[53] T. Mazzei, M. I. Cassetta, S. Fallani, S. Arrigucci, and A. Novelli, "Pharmacokinetic and pharmacodynamic aspects of antimicrobial agents for the treatment of uncomplicated urinary tract infections," International Journal of Antimicrobial Agents, vol. 28, supplement 1, pp. 35-41, 2006.

[54] F. Docobo-Pérez, G. L. Drusano, A. Johnson et al., "Pharmacodynamics of fosfomycin: insights into clinical use for antimicrobial resistance," Antimicrobial Agents and Chemotherapy, vol. 59, no. 9, pp. 5602-5610, 2015.

[55] N. Ceran, D. Mert, F. Y. Kocdogan et al., "A randomized comparative study of single-dose fosfomycin and 5-day ciprofloxacin in female patients with uncomplicated lower urinary tract infections," Journal of Infection and Chemotherapy, vol. 16, no. 6, pp. 424-430, 2010.

[56] J. L. Reffert and W. J. Smith, "Fosfomycin for the treatment of resistant gram-negative bacterial infections," Pharmacotherapy, vol. 34, no. 8, pp. 845-857, 2014.

[57] G. V. Sanchez, R. N. Master, J. A. Karlowsky, and J. M. Bordon, "In vitro antimicrobial resistance of urinary Escherichia coli isolates among U.S. outpatients from 2000 to 2010," Antimicrobial Agents and Chemotherapy, vol. 56, no. 4, pp. 2181-2183, 2012.

[58] A. Walkty, P. R. S. Lagacé-Wiens, J. A. Karlowsky et al., "Change in antimicrobial susceptibility of escherichia coli urinary tract isolates at a single institution over a period of 10 years," Canadian Journal of Microbiology, vol. 58, no. 3, pp. 345-349, 2012.

[59] Y. Doi, Y. S. Park, J. I. Rivera et al., "Community-associated extended-spectrum $\beta$-lactamase-producing Escherichia coli infection in the United States," Clinical Infectious Diseases, vol. 56, no. 5, pp. 641-648, 2013.

[60] L. Grigoryan, B. W. Trautner, and K. Gupta, "Diagnosis and management of urinary tract infections in the outpatient setting: a review," The Journal of the American Medical Association, vol. 312, no. 16, pp. 1677-1684, 2014.

[61] M. Grabe, R. Bartoletti, T. E. Bjerklund Johansen et al., Guidelines on Urological Infections, European Association of Urology, 2015.

[62] Anti-Infective Review Panel, Anti-Infective Guidelines for Community Acquired Infections, Anti-Infective Review Panel, 2014. 


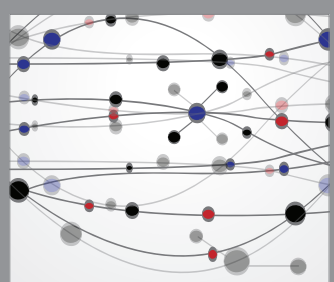

The Scientific World Journal
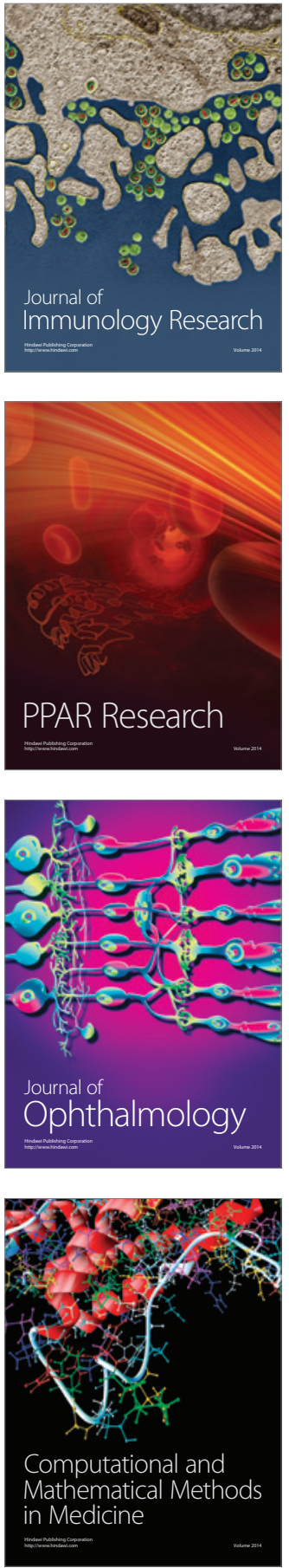

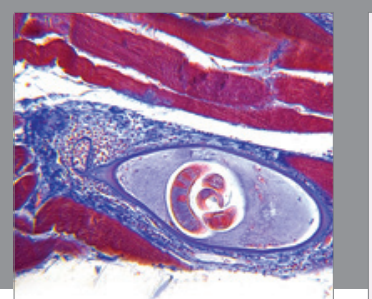

Gastroenterology Research and Practice

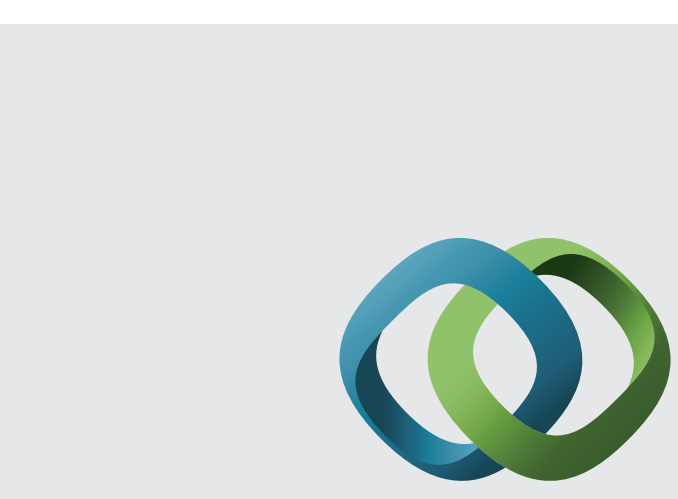

\section{Hindawi}

Submit your manuscripts at

http://www.hindawi.com
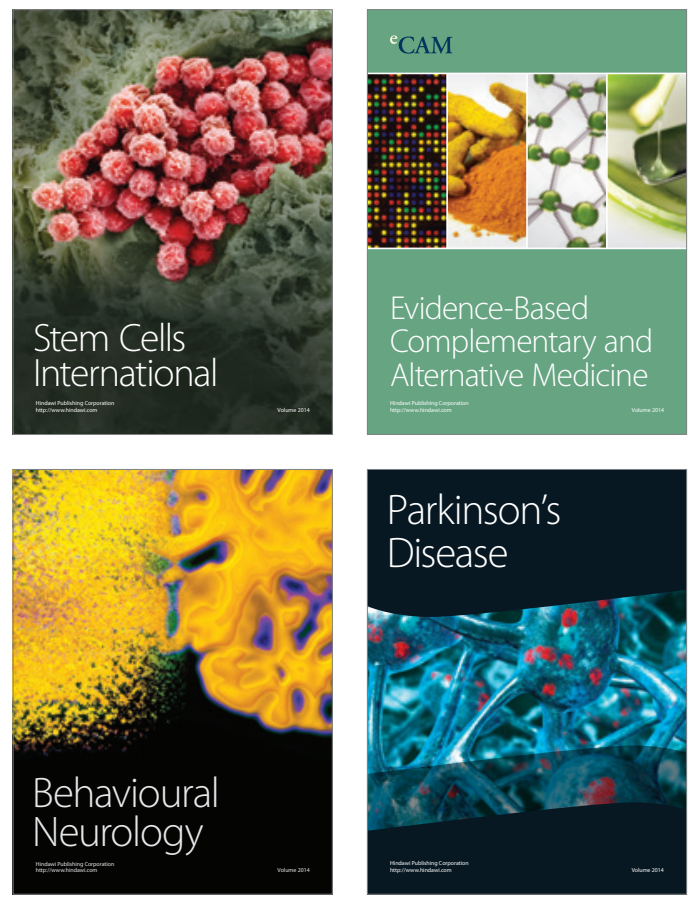
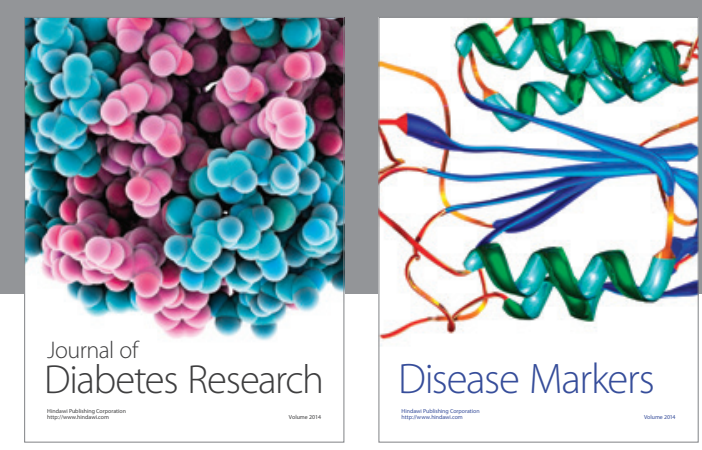

Disease Markers
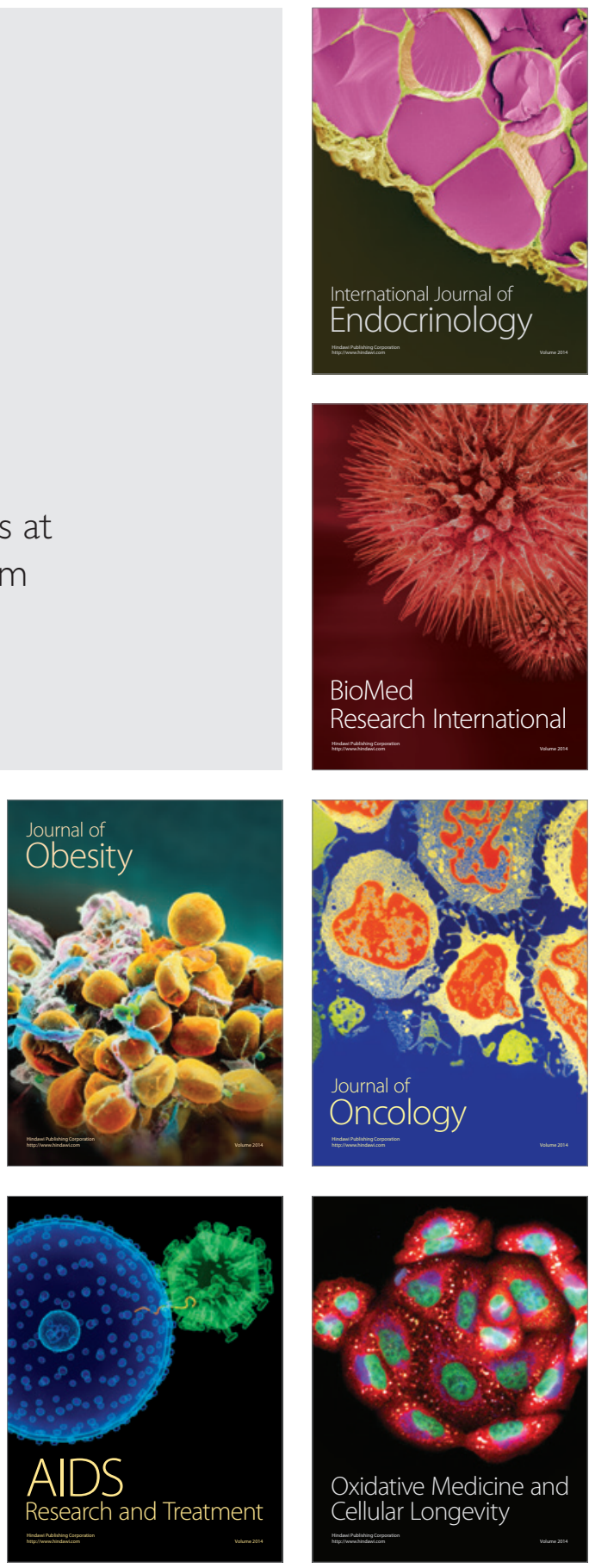